\title{
La mujer que mira Análisis de un «arrepentimiento» en la obra de Buñuel o donde Las Hurdes comienzan a ser Tierra sin pan
}




\section{Resumo}

Uno de los problemas que estaban sin resolver respecto a Las Hurdes (también conocida como Tierra sin pan) de Buñuel era precisamente saber las causas de esa doble denominación. A través del estudio de un plano -conocido como "La mujer que mira"- suprimido en la primera versión de 1933 (hasta ahora no conocida) y luego reinsertado en la versión definitiva de 1936 -inserción que cambia el sentido de toda la secuencia del "Descabezamiento de los gallos", la más importante y compleja de la película-, puede concluirse que la primera intención de Buñuel fue realizar un documental etnológico y realista sobre la comarca extremeña -de ahí Las Hurdes idea que va evolucionando hacia un planteamiento más universal y un lenguaje más personal y surrealista -de ahí Tierra sin pan - del que dicho “arrepentimiento" no sería sino su más elocuente expresión.

\section{Palabras clave}

Buñuel, la mujer que mira, Las Hurdes/Tierra sin Pan.

\section{Abstract}

This article has to do with one of the problem regarding the film Las Hurdes (also known as Tierra sin Pan). Such a problem concerns the causes justifying both titles. The "watching women" was a shot excluded in the first version of the movie and later it was included in the version of 1936 . The presence or absence of these filmic components interferes in the meaning: on one hand, ethnological content depends of the exclusion of the shot and, on the other hand, surrealistic significations depend on the inclusion of the shot. The author of the article discusses the creative relevance of this question.

\section{Keywords}

Buñuel, watching woman, Las Hurdes/Tierra sin Pan. 


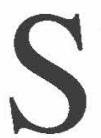
etenta y cinco años después de su realización Las HurdesTierra sin pan -su tercera película, considerada como uno de los mejores documentales de la historia del cine-aún presenta numerosas incógnitas, una de las cuales -si no la más importanteatañe precisamente a esa doble denominación pues mientras los primeros documentos coetáneos a la fecha de su primera versión (de 1933, prohibida como se sabe) casi siempre hablan de "Las Hurdes" sin embargo, tras el levantamiento de la prohibición y la realización de la segunda versión (1936) ya se habla de "Tierra sin pan" no sólo en España sino en Francia donde se estrena a finales de ese año con ese mismo título en francés. Las vicisitudes que median entre ambas fechas -y entre ambas versiones- han sido dadas a conocer por nosotros $^{1}$ en dos trabajos recientes apoyándonos en documentos inéditos y sobre todo en el análisis del material conservado de esa primera versión (dos bobinas) que hasta ese momento había permanecido inédito. De dicho análisis el aspecto más destacado y trascendental ha sido encontrarnos con un plano desechado de la primera versión y posteriormente rescatado para la segunda, operación que en nuestra opinión le da un giro copernicano a la película y la convierte en otra cosa diferente a lo que en principio iba a ser. Ese plano -al que denominamos "la mujer que mira" porque así aparece en el manuscrito original del comentario redactado ${ }^{2}$ por Pierre

1. Cfr. Estudios sobre Las Hurdes de Buñuel. Evidencia fílmica, estética y recepción. Sevilla: Renacimiento, 2006 y "El "bienio negro" de Buñuel y de Las HurdesTierra sin pan. Algunas aclaraciones a través de una correspondencia inédita". Boletín del Museo e Instituto Camón Aznar, 101 (2008), pp. 129-142

2. Filmoteca Española. Biblioteca, Archivo Buñuel 566 
Unik y el propio Buñuel- se encuentra dentro de la secuencia del "descabezamiento de los gallos" perteneciente a la parte rodada en La Alberca (Salamanca), que es con mucho la secuencia más elaborada y compleja de todas cuantas componen Las Hurdes-Tierra sin pan. He aquí el plano.

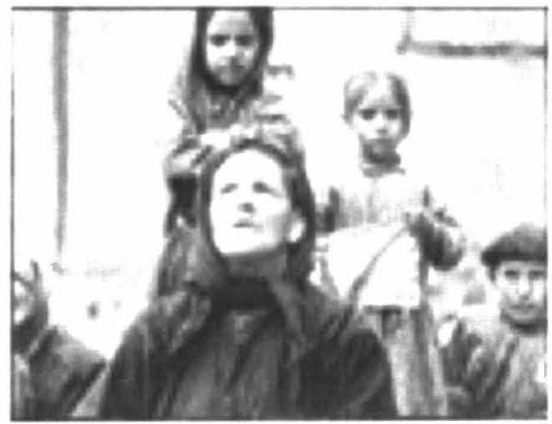

Y ahora su ausencia, es decir tal y como estaban planteados en el primer montaje los planos contigüos en las dos partes de la citada secuencia.

VERSION AUSENTE

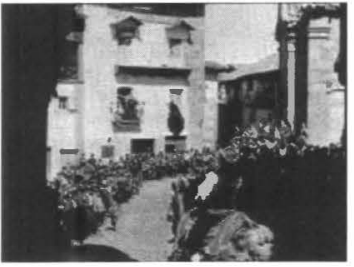
1933

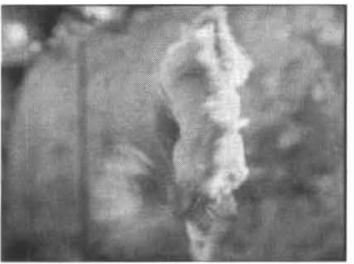

PLANOS $26-27$

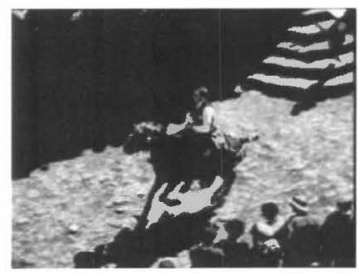

PLANOS 32.33

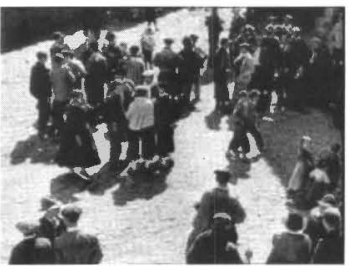


Ahora veamos cómo su inclusión entre los planos precedentes trastoca dicha relación de contiguiidad.

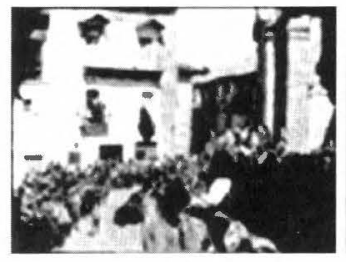

PLANO 27

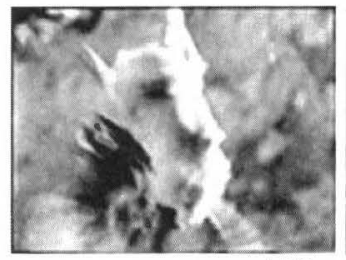

PLANO 35
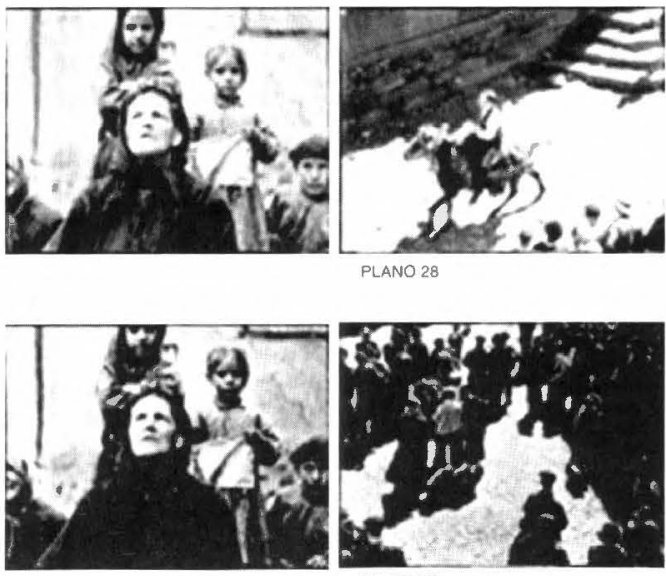

PLANO 36

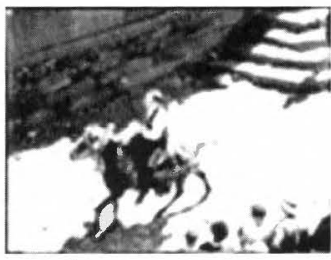

PLANO 28

Pero para hacernos una idea cabal de lo que supone tal "arrepentimiento" tenemos que analizar toda la secuencia en la que se inserta, comparando su ausencia en la versión de 1933 con su presencia en la de 1936.

Su desglose - con las líneas compositivas marcadas en rojo y las líneas de dirección del movimiento interno del plano en verde-es como sigue. 


\section{EL DESCABEZAMIENTO DE LOS GALLOS \\ SECUENCIA COMPLETA VERSION 1933 \\ COMPOSICION Y DIRECCION DEL MOVIMIENTO}
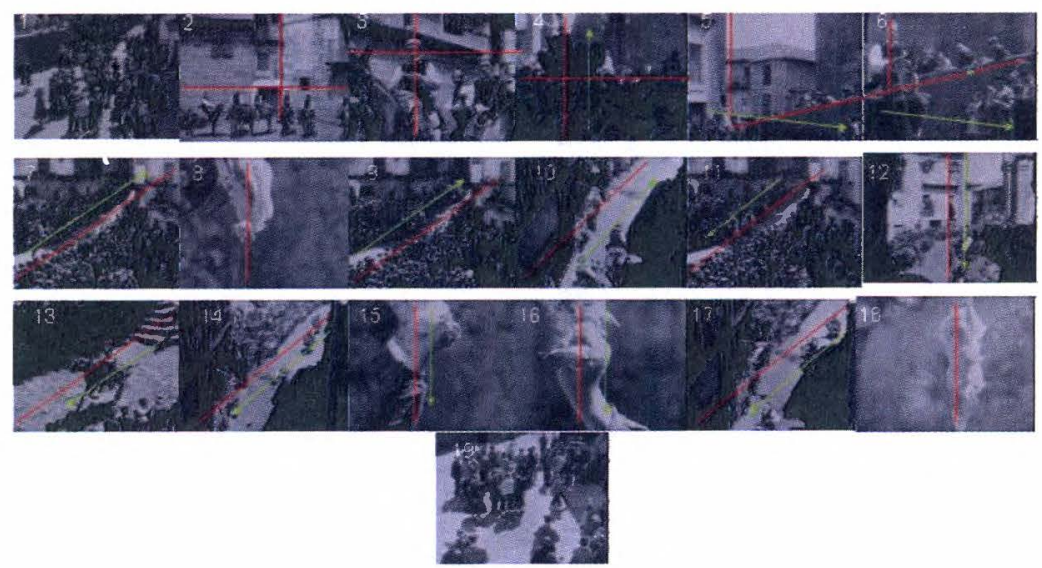

Como vemos consta en total 19 planos, de los cuales el primero y el último son simétricos y neutros. En los 17 restantes vemos una combinación general de planos estáticos (los que tienen una dominante horizontal o vertical o ambas a la vez, es decir planos equilibrados porque las líneas que definen a los sujetos y al fondo se mantienen en paralelo al marco) y planos con movimiento interno (en los que domina la línea oblicua y la composición en diagonal, cuya función suele ser romper el equilibrio de los otros). En este caso vemos que, tras una serie de planos estáticos con dominante verticalhorizontal (1-4), viene otra serie que, a través de las diagonales del movimiento de los caballistas (5-7), desestabiliza la composición, tendencia que al instante se rompe con la verticalidad y quietud del gallo cabeza abajo (8) e inmediatamente después reaparecer con otra serie de diagonales perfectas (9-11) -pues así se consideran las que terminan o nacen en el vértice del horizonte con el marco-, y a su vez de nuevo cortada por el «plano fuerte» de la secuencia (12), estático pero con un movimiento vertical de arriba-abajo (el arrancamiento de la cabeza del gallo) que rompe por su parte en un sentido dinámico y tensional la dominante anterior. Si nos fijamos el ritmo hasta ahora es cuaternario en tres series (1-4, 5-8, 9-12), en los 
que el plano cuarto de cada serie rompe con la cadencia anterior. A partir de ahora nos encontramos (13-16) con otra serie de ritmo igualmente cuaternario pero formado por dos binarios enfrentados entre sí, regular y simétricamente en sus movimientos y sus direcciones (13-14 versus 15-16), para concluir en un ternario con tres planos simples (17-19) de la misma naturaleza que la oposición anterior, de los cuales el último (19) -neutro- concuerda simétricamente con el primero. Así pues: la secuencia está perfectamente estudiada y planificada para producir un efecto de carácter expositivo-descriptivo según el modo clásico de representación, proporcionado y simétrico, sin altibajos y sin grandes contrastes (tenemos por ejemplo igualdad de planos estáticos y móviles), donde el respeto al tema es el factor esencial, pero acaso por todo ello, el ritmo resulta regular y repetitivo, con tendencia a la monotonía, en exceso lineal y por ello de lectura superficial.

Sin embargo, en la segunda versión -tal y como vemos a continuación en su desglose- tenemos que consta de 5 planos más, lo que es indicio de una mayor complejidad compositiva.

\section{EL DESCABEZAMIENTO DE LOS GALLOS SECUENCIA COMPLETA VERSION 1936 (1965) COMPOSICION Y DIRECCION DEL MOVIMIENTO}
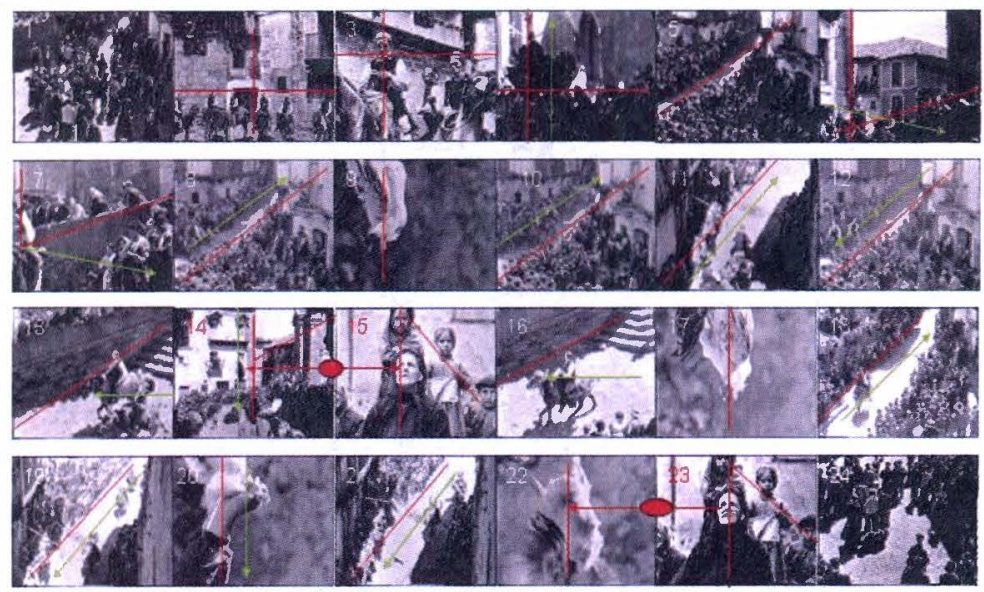
Si al comienzo coincide con la de 1933 en el ritmo cuaternario y los planos estáticos con dominante vertical-horizontal, enseguida (5) introduce una novedad: un plano estático de la escena donde se van a desarrollar los planos móviles siguientes (6-8) con la característica composición en diagonal, hecho que cambia el sesgo del ritmo hacia un sistema quinario cuya última unidad (9) -el gallo colgando cabeza abajo- tiene, como en los casos vistos anteriormente, un efecto congelante por su verticalidad, aunque de inmediato provoca otra nueva serie quinaria (10-14) de planos móviles con direcciones contrapuestas que culmina (14) con otro plano estático -el «plano fuerte» del descabezamiento del gallo- tras el cual -y ahí viene la gran novedad- se incorpora por primera vez «la mujer que mira», que va a romper radicalmente todo el discurso y el sentido de la película. Pero recordemos lo que había cuando la mujer estaba ausente; era esto
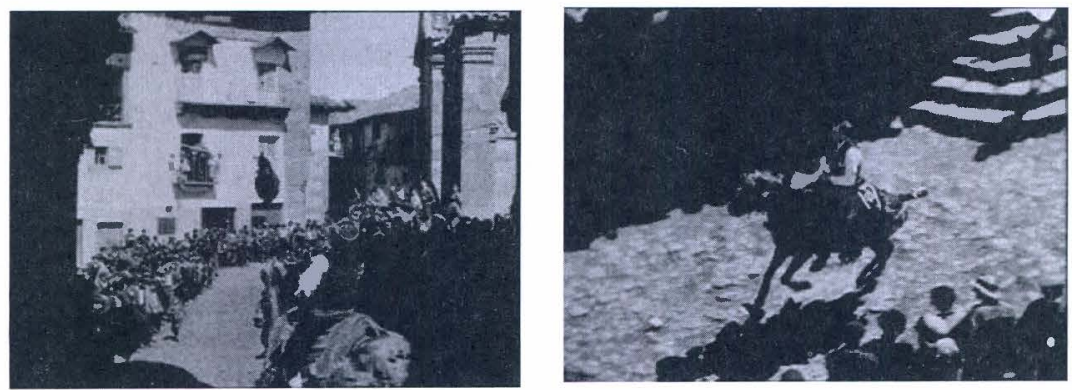

un plano estático -el fuerte- que centra toda la acción y después un caballista en dirección derecha-izquierda como si no tuviera que ver nada con lo que antecede: hay una simple conjunción de planos sin ningún vínculo formal entre sí, es decir: el sistema expositivo propio del documental clásico. Su inclusión -veámoslo de nuevo- le đa un 


\section{LA MUJER QUE MIRA \\ DETALLE \\ DIRECCION DE LA MIRADA Y EJE DE LA ACCION}

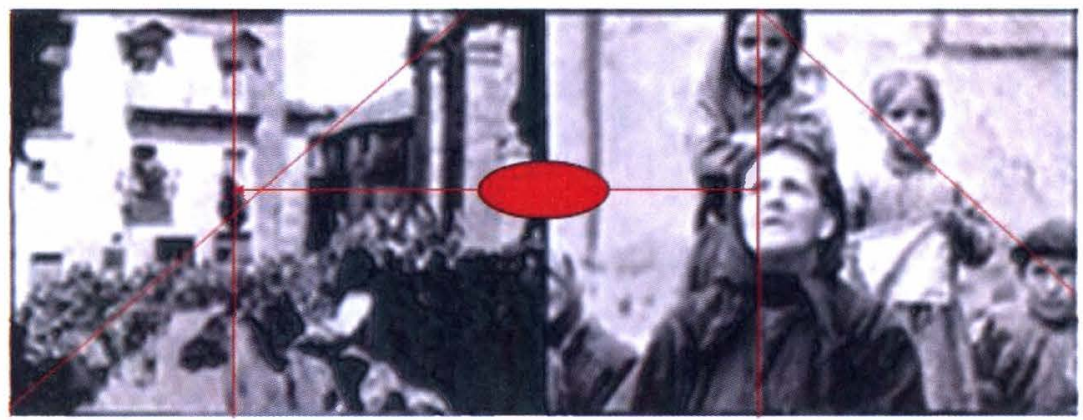

nuevo sentido al plano precedente reforzando si cabe aún más su carácter de «plano fuerte» al acentuar su estatismo, un plano estático -el fuerte- que centra toda la acción y después un un plano estático -el fuerte-que centra toda la acción y después caballista en dirección derecha-izquierda como si no tuviera que ver nada con lo que antecede: hay una simple conjunción de planos sin ningún vínculo formal entre sí, es decir: el sistema expositivo propio del documental clásico. Su inclusión -veámoslo de nuevo- le da un nuevo sentido al plano precedente reforzando si cabe aún más su carácter de «plano fuerte» al acentuar su estatismo, su verticalidad y su fuerza descendente, pero al mismo tiempo introduce otros elementos nuevos que van a resultar decisivos:

- frente a la ausencia de movimiento interno y de su correspondiente dirección introduce un movimiento de carácter más sutil: la dirección de la mirada interna que se dirige desde la mujer como espectadora del suceso hasta el «punto fuerte» de la acción: el gallo colgado de la soga, que está a punto de ser descabezado por el caballista; 
- la composición en diagonal propia de los planos móviles y desequilibrantes se refuerza, pero de una manera estática por medio del alineamiento decreciente de los niños que están detrás de la mujer, diagonales que tienden a confluir en un punto fuera del plano y que por ello alcanzan en este caso el nivel más alto de creatividad que puede lograrse con este tipo de recurso;

- la dirección de la mirada interna coincide exactamente con el «eje de la acción» que nos conecta a nosotros como espectadores dentro del cubo imaginario en el que nos encontramos viendo la película: primero con el gallo y después con los ojos de la mujer que están mirando a su vez precisamente al gallo;

- ese vínculo comunicativo con nosotros espectadores se acentúa aún más -y de un modo inconsciente, como le gustaba a Buñuel- a través de la mirada directa de las dos niñas, que están detrás de la mujer, hacia la cámara, es decir hacia nosotros: miradas que no podemos obviar y por las que nos sentimos -sin darnos cuenta- atrapados en la urdimbre del director, cuyo objetivo no es otro que -como el caballista al gallo, como el navajazo al ojo en Un perro andaluz- arrancarnos de cuajo la cabeza.

Para decirlo con otras palabras: al arrepentirse de cómo quedaba la secuencia en la primera versión -seguramente tras los trágicos sucesos de Asturias y el visionado negativo de Marañón- y al decidirse a incluir este plano definitivamente, Buñuel -a través de la mujer, utilizada a modo de señuelo- cambia su mirada hacia la "representación" de la realidad que tiene delante en la moviola, desiste de verla en sus aspectos de "realidad" que reproduce fielmente una fiesta sanguinaria y cruel, y resuelve llevar al espectador a su terreno ficcional e implicarle sin que tenga clara conciencia de lo que ve. 


\section{EL DESCABEZAMIENTO DE LOS GALLOS SECUENCIA COMPLETA VERSION 1936 (1965) COMPOSICION Y DIRECCION DEL MOVIMIENTO}
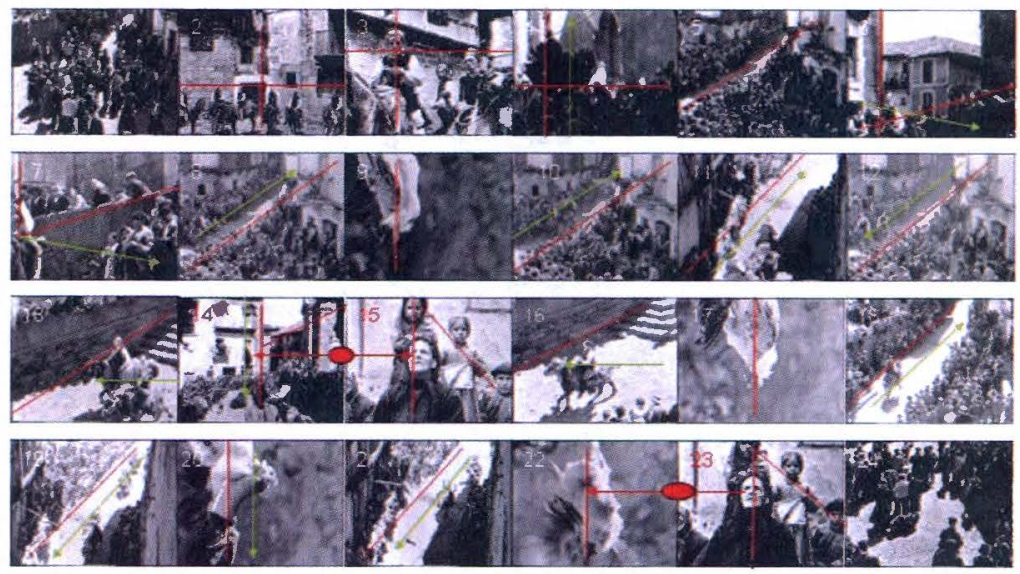

Tras este "choque" frontal (volvemos a la secuencia), que supone la aparición de la mujer que mira y de los niños que nos miran, la película ya no puede ser la misma; y en efecto el ritmo se desequilibra -se desboca- y ya no encontramos una cadencia regular ni proporcionada ni simétrica: véase si no cómo el plano 16 parece continuación del 13, como si la secuencia volviera a empezar, y cómo a partir de ahí (17-21) el contraste entre verticales y diagonales e incluso entre direcciones no sigue un ritmo acompasado sino que parece todo dirigirse inexorablemente al estallido final (22-23) donde vuelve a reaparecer la mujer.

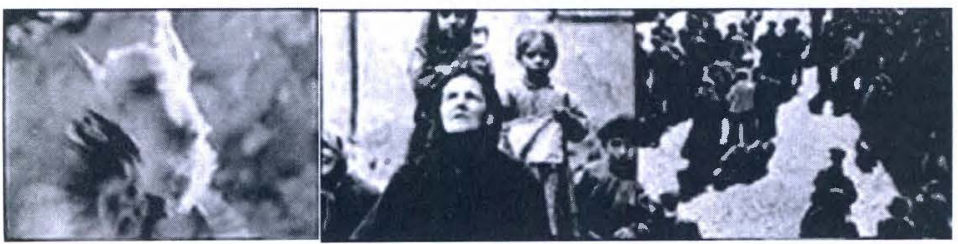


Pero en esta ocasión, superado ya el «choque emocional», el fuerte impacto de esas miradas que miran y nos miran, se trata tan sólo de que tengamos la dosis de recuerdo, la que nos certifica nuestra defunción: el gallo revolotea suspendido en el aire ya sin cabeza y no hay remedio; sólo nos queda dejarnos llevar -si antes (con Un perro andaluz) nos hemos quedado ciegos y somos invitados a ver de otra forma, ahora hemos sido decapitados... ya no podremos ni pensar porque sencilla y metafóricamente hemos dejado de existir...

Pero no nos desesperemos, que es ahí donde empieza la función, donde el documento se convierte en arte, donde la ficción supera a la realidad, donde el cine no deja de ser cine y donde hasta la tragedia, el hambre, la miseria, la muerte incluso pueden tener algo de cómico... y si no se lo creen veamos a esa niña que hay detrás de la mujer que abre la boca de asombro ante la salvajada que acaba de presenciar; la niña "realmente" se ríe a carcajada limpia o a mandíbula batiente (como decimos en español castizo) pero es que su carcajada se dirige a nosotros - a la cámara - y en su espontaneidad nos delata el engaño en el que estamos sumidos, la esencial mascarada de todo arte o la simulación que es en sí toda película, pues nadie diría que se encuentra asistiendo a un espectáculo cruel y bárbaro, antesala de todos los horrores que vendrán después y que el espectador decapitado verá -no sabemos cómo- referidos a la comarca hurdana, ya que es aquí, justamente en este plano, donde Las Hurdes, por obra y gracia de un ser "arrepentido", ha dejado de ser un documental artísticopintoresco para convertirse simplemente en Tierra sin pan, uno de los mejores ¿documentales? de la historia del cine, la tercera película en la filmografía de un director único: Luis Buñuel. 
LA MUJER QUE MIRA

ULTIMO FOTOGRAMA DEL PLANO 23

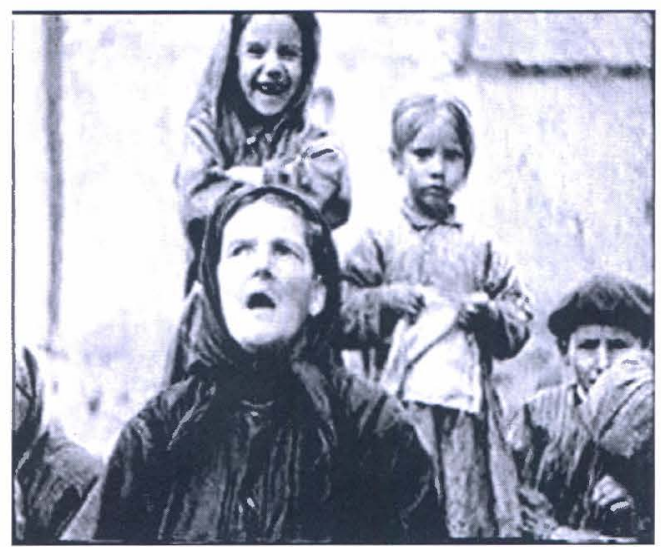

\section{Bibliografia}

BUÑUEL, Luis. 1998. Land without Bread. Nickel Odeón, número 13

FUENTES, Víctor. 2005. La mirada de Buñuel. Cine, Literatura y Vida. Madrid: Tabla Rasa.

SÁNCHEZ VIDAL, Agustín. 1999. De las Hurdes a Tierras sin Pan, in Javier (Comis.)

Las Hurdes, un documental de Luis Buñuel. Badajoz: Museo Extremeño e Iberocamericano de Arte, pp.38-75.uerdeerrera Comis.),

TURRENT,Tomás Pérez y COLINA, José de la Colina. 1993. Buñuel por Buñuel. Madrid: Plot. 